\title{
Transient Absorption of DNA bases in the gas phase and in chloroform solution: a comparative quantum mechanical study ${ }^{\dagger}$
}

\author{
Daniil A. Fedotov, ${ }^{a}$ Alexander C. Paul, ${ }^{b}$ Henrik Koch, ${ }^{b, c}$ Fabrizio Santoro, ${ }^{d}$ Sonia \\ Coriani, ${ }^{a, b}$ and Roberto Improta ${ }^{e}$ \\ We study the excited state absorption (ESA) properties of the four DNA bases (thymine, cytosine, \\ adenine, and guanine) by different single reference quantum mechanical methods, i.e. equation \\ of motion coupled cluster singles and doubles (EOM-CCSD), singles, doubles and perturbative \\ triples (EOM-CC3), and time-dependent density functional theory (TD-DFT), with the long-range \\ corrected CAM-B3LYP functional. Preliminary results at the Tamm-Dancoff (TDA) CAM-B3LYP \\ level using the maximum overlap method (MOM) are reported for Thymine. In the gas phase, \\ the three methods predict similar One Photon Absorption (OPA) spectra, which are also consistent \\ with the experimental results and with the most accurate computational studies available in the \\ literature. The ESA spectra are then computed for the $\pi \pi^{*}$ states (one for pyrimidine, two for \\ purines) associated with the lowest energy absorption band, and for the close lying $\mathrm{n} \pi^{*}$ state. The \\ EOM-CC3, EOM-CCSD and CAM-B3LYP methods provide similar ESA spectral patterns, which are \\ also in qualitatively agreement with literature RASPT2 results. Once validated in the gas phase, \\ TD-CAM-B3LYP has been used to compute the ESA in chloroform, including solvent effect by the \\ polarizable continuum model (PCM). The predicted OPA and ESA spectra in chloroform are very \\ similar to those in the gas phase, most of the bands shifting by less than $0.1 \mathrm{eV}$, with a small \\ increase of the intensities and a moderate destabilization of the $\mathrm{n} \pi^{*}$ state. Finally, ESA spectra \\ have been computed from the minima of the lowest energy $\pi \pi^{*}$ state, and are consistent with the \\ available experimental transient absorption spectra of the nucleosides in solution, providing a final \\ validation of our computational approach.
}

\section{Introduction}

Pump-probe spectroscopy is the key tool to investigate fast photoinduced dynamics. ${ }^{1-3}$ In transient absorption experiments, the excited state prepared by the pump pulse can further absorb the probe pulse, a process known as excited state absorption (ESA), which, together with the ground state bleaching and the stimulated emission, determines the observed signal. ${ }^{1,2}$ Each excited electronic state has its characteristic ESA spectrum, making the correct interpretation of this phenomenon fundamental to disentangle the photoactivated dynamics. ${ }^{1,2}$ This is a quite challenging task due to the large congestion of the excited electronic states in the high energy region and, at the same time, to the interference' of emission and ground state absorption processes, making the contribution of quantum mechanical calculations crucial. 4-14 In this respect, in a very recent study ${ }^{15}$ we considered the two

${ }^{a}$ DTU Chemistry, Technical University of Denmark, 2800 Kongens Lyngby, Denmark. E-mail: soco@kemi.dtu.dk

${ }^{b}$ Department of Chemistry, NTNU - Norwegian University of Science and Technology, N-7491 Trondheim, Norway

'Scuola Normale Superiore, Piazza dei Cavalieri, 7, I-56126, Pisa, Italy. E-mail: henrik.koch@sns.it

${ }^{d}$ Istituto di Chimica dei Composti Organometallici (ICCOM-CNR), Area della Ricerca del CNR, I-56124 Pisa, Italy. E-mail: fabrizio.santoro@iccom.cnr.it

${ }^{e}$ Istituto di Biostrutture e Bioimmagini-CNR, I-80134 Napoli, Italy; E-mail: robimp@unina.it

$\dagger$ Electronic Supplementary Information (ESI) available lowest excited states of uracil and benchmarked the ESA spectra computed by TD-DFT and the widely used CAM-B3LYP functional, ${ }^{16}$ with those provided by some accurate wavefunctionbased methods, namely equation of motion coupled cluster singles and doubles (EOM-CCSD), ${ }^{17}$ singles, doubles and perturbative triples (EOM-CC3) ${ }^{18}$ and three methods of the Algebraic Diagrammatic Construction family, namely ADC(2), ADC(2)-X and $\operatorname{ADC}(3 / 2){ }^{19}$

In this study, we take further steps towards a full assessment of the performance of different single reference electronic structure methods in calculations of the ESA spectra. On the one hand, we extend our comparative analysis to all DNA bases: thymine, cytosine, adenine, and guanine (see Figure 1). For these species, we compute OPA and ESA spectra at the Franck-Condon (FC) point, for the first (or two first) lowest-energy $\pi \pi^{*}$ states plus the lowestenergy $n \pi^{*}$ state, in gas phase. In this first task, we compare the predictions of TD-DFT/CAM-B3LYP, EOM-CCSD, and EOM-CC3. At least for the lowest-energy $\pi \pi^{*}$ states, the results of a recent RASPT2 study ${ }^{13}$ will provide an additional useful check, especially for what concerns the possible effect of double excitations. Using thymine as test case, we also carry out exploratory ESA calculations using the MOM-TDA approach. The ESA spectra are in this case obtained by computing, at CAMB3LYP-TDA level, the OPA of non-aufbau solutions of the Kohn-Sham equations corresponding to the dominant Molecular Orbital (MO) excitation in the excited states of interest. These higher energy KS solutions can be interpreted as single-determinant approximations to the 
excited states of the system and are optimized using the MOM approach. ${ }^{20}$ MOM-TDDFT (and even MOM-CCSD) is often used to obtain $\mathrm{x}$-ray absorption spectra of valence excited states, i.e. to simulate valence pump-core probe spectra. ${ }^{21-24}$ We are not aware of previous studies applying this approach for ESA in the UV-vis region.

Once assessed the accuracy of CAM-B3LYP, we use this method to compute the ESA for all the bases in chloroform solution, simulated by means of the polarizable continuum model (PCM). ${ }^{25} \mathrm{Fi}-$ nally, we compute the ESA in chloroform from the minima of the lowest-energy bright states, in order to allow a a more direct comparison between our predictions and the available experimental spectra.

We selected the DNA bases for two different reasons. On one side, they are fairly complex heterocyclic molecules, pyrimidines (thymine and cytosine) and purines (adenine and guanine) with exocyclic substituents (carbonyl and amino groups) strongly coupled with the $\pi$ ring. As a consequence, several excited states with different character $\left(\pi \pi^{*}, \mathrm{n} \pi^{*}, \pi \sigma^{*}\right.$, and Rydberg states) lay close in energy in the FC region. ${ }^{26}$ They constitute therefore challenging, and, at the same time, probative test cases. On the other side, the photoactivated dynamics of nucleobases is of great biological relevance, since absorption of UV light by DNA can trigger many potentially dangerous oxidative processes. ${ }^{27-29}$ For this reason, many time-resolved experiments and computational studies are available for nucleobases, providing extremely useful data for any comparative analysis. ${ }^{26,30-35}$

\section{Computational details}

$C_{S}$-symmetry structures of all molecules were optimised at the CAM-B3LYP/6-311+G(d,p) level of theory (Fig. 1). In the following they will be referred to as the FC point. TD-DFT calculations using the CAM-B3LYP functional were carried out with Dalton. ${ }^{36}$ The EOM-CCSD and EOM-CC3 calculations in gas phase were performed using $e^{T}{ }^{37}$ The aug-cc-pVDZ basis set was used in all cases. The spectra in chloroform solution were obtained applying $\mathrm{PCM}^{25}$ in chloroform, without any further geometry optimization. TD-DFT calculations in the Tamm-Dancoff approximation (TDA) were run with Turbomole ${ }^{38}$ and Q-Chem. ${ }^{39}$ The MOM-TDA ESA calculations on thymine were performed with QChem. ${ }^{39}$ The results are reported in the ESI, Figure S16, and briefly discussed in the following section.

Tables reporting the TD-DFT, EOM-CCSD and EOM-CC3 OPA and ESA (energies and oscillator strengths) for each electronic transition are given in the ESI. The first ionization energy (IE) of the ground state was obtained at the EOM-CCSD and EOMCC3 level as excitation into a bath orbital. ${ }^{40-42}$ For CAM-B3LYP, we computed the IE as difference between the total energy of the cation and the total energy of the neutral at the FC geometry $(\triangle \mathrm{SCF})$. Estimates of the first ionization energy of the excited states were calculated as difference between the first IE of the ground state and the excitation energy of the valence excited state of the interest, according to the vertical approximation. A summary of IEs for all systems is presented in ESI in Table S1. For a characterization of the relevant excited states in terms of natural transition orbitals (NTO), see Figure S1.
To facilitate the discussion, we report in Figures S2-S5 in ESI a comparison of our spectra with spectra constructed from the RASPT2/ANO-L energies and oscillator strengths of ref. 13.

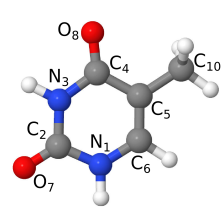

(a)

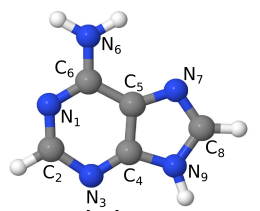

(C)

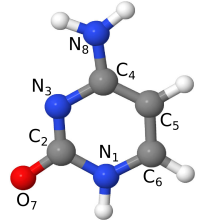

(b)

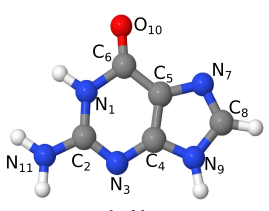

(d)
Fig. 1 The molecules considered in the study: (a) thymine; (b) cytosine; (c) adenine; (d) guanine.

As detailed in Table S2, for thymine and cytosine at least 40 excited states have been included in the ESA calculations for all the methods considered. This enabled us to obtain spectra up to $4 \mathrm{eV}$. For adenine and guanine, at the EOM-CC3 and EOM-CCSD level it was possible to include only a smaller number of states. As a consequence, the computed spectra cover a smaller energy region, i.e. up to $1 \sim 1.8 \mathrm{eV}$ for EOM-CC3, up to $2 \sim 2.8 \mathrm{eV}$ for EOM-CCSD, and up to $\sim 3 \mathrm{eV}$ for CAM-B3LYP.

\section{Results}

\subsection{Thymine}

Figure 2 shows that the OPA spectra computed in the gas phase by CAM-B3LYP and EOM-CC3 are fairly similar. They exhibit two bands slightly above $5 \mathrm{eV}$ and at $\sim 6.5 \mathrm{eV}$, with similar intensity, followed by a more intense one at $\sim 7.8 \mathrm{eV}$. The EOM-CCSD spectrum has a similar shape, with a uniform blue-shift of $\sim 0.2 \mathrm{eV}$. The RASPT2 spectrum is also similar to the CAM-B3LYP one, but for a small uniform red-shift. ${ }^{13}$ All the spectra are consistent with the available experimental ones (see Refs. 43 and 13 for a discussion). The EOM-CC3 peaks are blue shifted by $\sim 0.25 \mathrm{eV}$ with respect to the experiments, ${ }^{44}$ a value which is is expected to be largely due to the absence of vibronic effects in the present calculation. ${ }^{43,45}$ The lowest energy band is associated to a $\pi \pi^{*}$ state, with $\mathrm{HOMO} \rightarrow$ LUMO character, though in the gas phase the lowest energy excited state is a $\mathrm{n} \pi^{*}$ state. This picture is very similar to the one we analyzed in detail in our previous study on uracil. ${ }^{15}$

Inclusion of solvent effects has a very modest influence on the OPA spectrum, apart from a general increase of the intensities, likely due to the linear response implementation of PCM in TDDFT. ${ }^{46,47}$ The most significant consequence is the destabilization (by $\sim 0.23 \mathrm{eV}$ ) of the $\mathrm{n} \pi^{*}$ state, confirming a trend already evidenced in the literature. ${ }^{26,48}$ As a consequence, the $n \pi^{*}$ state is $S_{1}$ in the gas phase and $S_{2}$ in chloroform.

We notice that EOM-CC3 and CAM-B3LYP predict similar ESA spectra for the lowest-energy bright state, the former being, on 


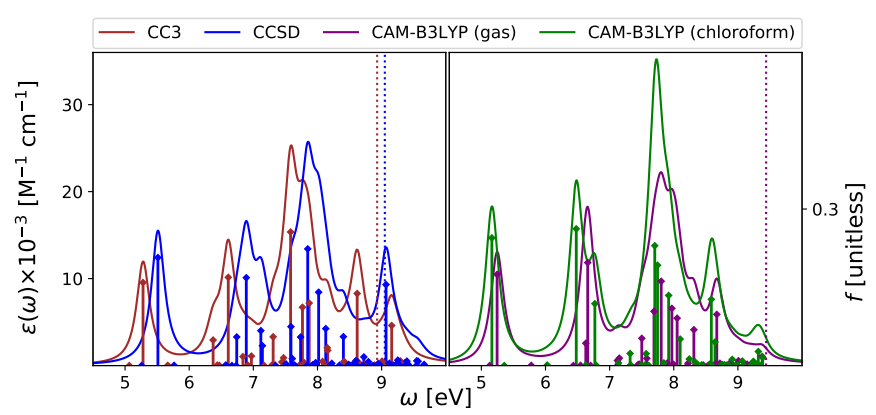

Fig. 2 Thymine. Left: OPA at EOM-CCSD/aug-cc-pVDZ and EOMCC3/aug-cc-pVDZ levels of theory in gas phase. Right: OPA at CAMB3LYP/aug-cc-pVDZ level of theory in gas phase and in chloroform solution. The vertical lines indicate the first ionisation energy in the gas phase ( $\triangle \mathrm{SCF}$ at DFT level).

the average, slightly red-shifted. We observe a broad absorption band in the $1 \sim 2.5 \mathrm{eV}$ range, with a prominent peak at $\sim 1.5 \mathrm{eV}$ and another, smaller, at $\sim 2 \mathrm{eV}$. Then, two very large peaks are predicted at $\sim 3$ and $\sim 3.5 \mathrm{eV}$. On balance, the EOM-CCSD spectrum is also similar to the EOM-CC3 one.

Overall, these spectra are reasonably consistent with the predictions of RASPT2, considering that, due to the selection of the active space and the basis set employed there, the number of excited states in RASPT2 is smaller. ${ }^{13}$ Indeed, according to RASPT2, the ESA spectrum is made up of two peaks at 1.2 and $1.5 \mathrm{eV}$, a smaller one at $\sim 2 \mathrm{eV}$, and two more intense ESA transition at $\sim 3$ and $\sim 3.5 \mathrm{eV},{ }^{13}$ see also Figure S2.

The ESA spectrum computed for the lowest energy $\mathrm{n} \pi^{*}$ state in the gas phase is rather similar to the one predicted for uracil, ${ }^{15}$ with two weak bands peaking at $\sim 2 \mathrm{eV}$ and $\sim 4 \mathrm{eV}$. Also in this case, EOM-CC3 and CAM-B3LYP spectra are very similar, while according to EOM-CCSD the lowest energy band is blue shifted by $\sim 0.5 \mathrm{eV}$.

Like for OPA, inclusion of solvent effect has limited effect on the computed ESA, which is dominated by transitions between states with the same symmetry. As a consequence, the ESA spectra computed in chloroform are very similar to those obtained in the gas phase, but some differences appear. For example, the lowest energy ESA band exhibits a small, but well visible, redshift in chloroform. This is due to the small separation induced by the solvent between $2 \mathrm{~A}^{\prime}$ and $3 \mathrm{~A}^{\prime}$ transitions, which are instead almost iso-energetic in the gas phase. A more important effect is observed for the second ESA band of the $n \pi^{*}$ state, which is red-shifted by $\sim 0.6 \mathrm{eV}$ in chloroform.

To conclude this subsection, we briefly comment on TDA versus TD-DFT for the OPA spectrum, as well as on MOM-TDA versus regular TDA quadratic response for ESA. With reference to Figure S16, TDA and TD-DFT yield similar spectral shape for the OPA and ESA spectra, the main difference is a small blue shift in energy, and a more peaked shape of the intense band at around $8 \mathrm{eV}$. The TDA ESA spectra obtained from the MOM-optimized $1 n$ and $1 \pi$ states are more at variance from those yielded by TDA quadratic response, as even more blue-shifted compared to TDDFT. Even though the intensities are of comparable size, noticeable intensity redistribution is moreover observed between the peaks. Given these results, and keeping in mind that the final excited states obtained from MOM-TDA are significantly spincontaminated, it is unclear whether MOM-TDA linear response is a valuable alternative to quadratic response TDA/TD-DFT to obtain ESA spectra. Further studies are needed.

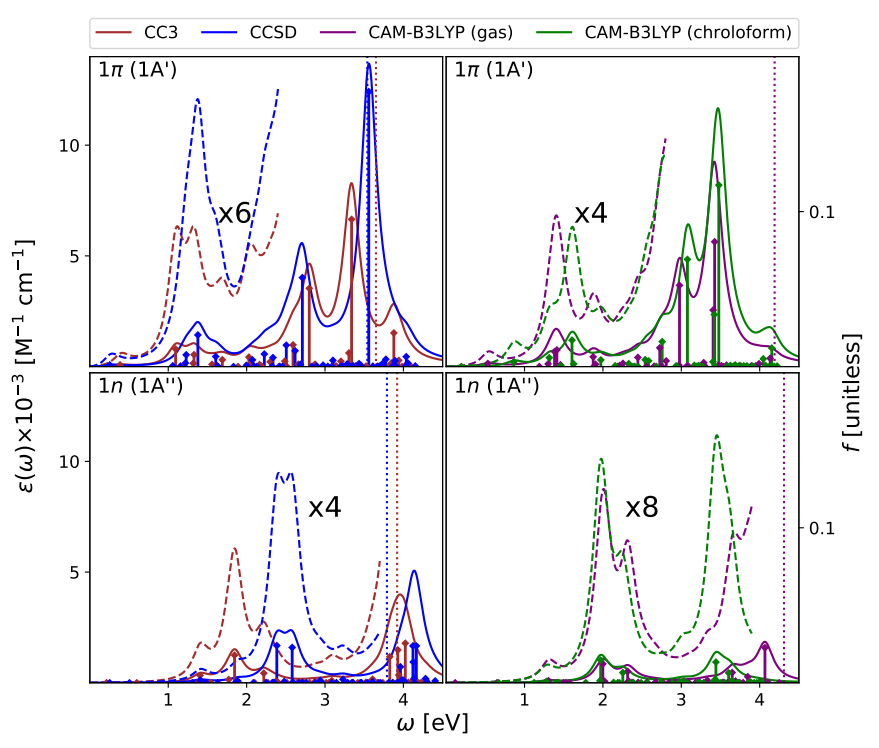

Fig. 3 Thymine. Left: ESA at EOM-CCSD/aug-cc-pVDZ and EOMCC3/aug-cc-pVDZ levels of theory in gas phase. Right: ESA at CAMB3LYP/aug-cc-pVDZ level of theory in gas phase and in chloroform solution. The spectra in dashed line are enhanced by the factor given in the figures. The vertical lines indicate the first ionisation energy in the gas phase (see Section 2 for details).

\subsection{Cytosine}

In the case of cytosine, we focused our analysis on the keto-amino tautomer, which is the most stable in condensed phase ${ }^{11}$ and the one present within DNA. Note, however, that other tautomers are more stable in gas phase, ${ }^{49,50}$ and they have to be included when comparing the experimental and the computed OPA spectra. ${ }^{50}$

In Figure 4 we report the OPA spectra computed with EOMCC3 and EOM-CCSD in the gas phase, and at the TD-CAM-B3LYP level both in the gas phase and in chloroform. EOM-CC3 predicts four prominent peaks below $7 \mathrm{eV}$, and, after a broad and rather structureless absorption band, another peak just above 8 eV. EOM-CCSD and TD-CAM-B3LYP provide very similar spectral patterns, for what concerns the relative energy and intensity of the main peaks, but the spectra are almost uniformly shifted by $0.2 \sim 0.3 \mathrm{eV}$. A detailed description of the lowest energy excited states of cytosine can be found in Refs. 43,51. The $S_{1}$ state is a $\pi \pi^{*}$ transition, with predominant $\mathrm{HOMO} \rightarrow$ LUMO character, while the $S_{2} n \pi^{*}$ state involves the excitation from the lone pair of the nitrogen in position 3 towards the LUMO. The three methods applied in this study provide OPA spectral shapes very similar to those obtained by RASPT2/ANO-L, which are in almost quantitative agreement with EOM-CC3 ${ }^{13}$ apart from a more intense central peak and a slight shift of the band at $8 \mathrm{eV}$, see Figure S3. 
Inclusion of solvent effects has the same, small, impact on the OPA spectrum, as we have already discussed for Thy - that is, a general increase of the intensities and a destabilization (by $\sim 0.3$ $\mathrm{eV}$ ) of the $\mathrm{n} \pi^{*}$ states.

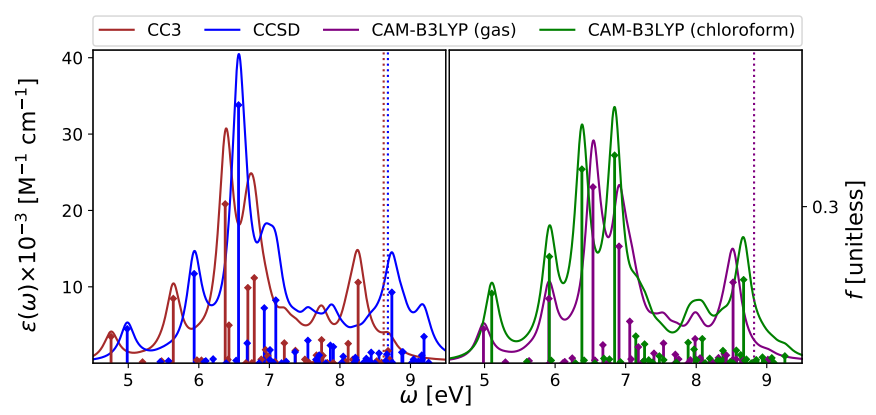

Fig. 4 Cytosine. Left: OPA at EOM-CCSD/aug-cc-pVDZ and EOM-CC3/aug-cc-pVDZ levels in gas phase. Right: OPA at CAMB3LYP/aug-cc-pVDZ level of theory in gas phase and in chloroform solution. The vertical lines indicate the first ionisation energy in the gas phase ( $\triangle$ SCF at DFT level).

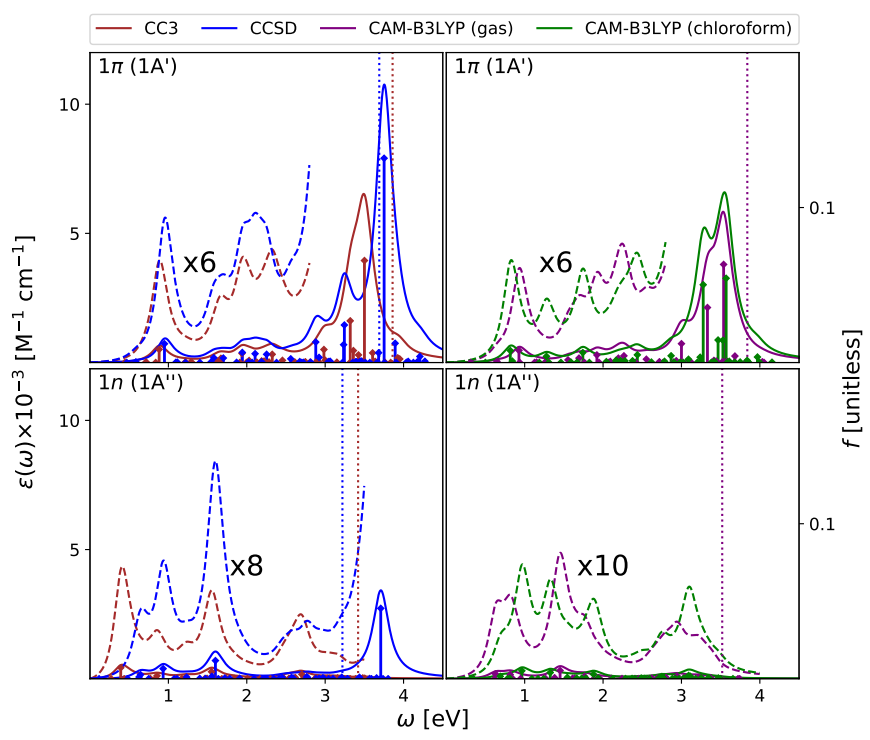

Fig. 5 Cytosine. Left: ESA at EOM-CCSD/aug-cc-pVDZ and EOMCC3/aug-cc-pVDZ levels of theory in gas phase. Right: ESA at TDCAM-B3LYP/aug-cc-pVDZ level of theory in gas phase and in chloroform solution. The spectra in dashed line have been enhanced by the factor given in the figure. The vertical lines indicate the first ionisation energy in the gas phase ( $\triangle \mathrm{SCF}$ at DFT level).

In Figure 5 we report the ESA computed at the same level of theory as the OPA. In the gas phase, the three methods predict quite similar ESA spectra for the lowest energy $\pi \pi^{*}$ state. We observe a first peak just below $1 \mathrm{eV}$ and another broad band centered at $\sim 2 \mathrm{eV}$. However, the presence of many very weak transitions, likely associated to Rydberg states, makes the entire spectrum below $3 \mathrm{eV}$ very congested and not well resolved. After a peak at $\sim 3 \mathrm{eV}$, at $\sim 3.5 \mathrm{eV}$ we then find two intense transitions, which give rise to a very strong band. The main quantitative dif- ference between the three spectra is a blue-shift of the most intense peak predicted by EOM-CCSD. These spectra are consistent with those computed at the RASPT2/ANO-L level (see Figure S3), especially when considering that in this latter study only $\pi \pi^{*}$ transitions are included. ${ }^{13}$ At this latter level of theory, after two weak transitions at $\sim 0.9 \mathrm{eV}$ and $\sim 2.2 \mathrm{eV}$, a strong peak at $3.35 \mathrm{eV}$ is found.

The ESA spectrum of the lowest energy $n \pi^{*}$ state is generally weaker than the one of the $\pi \pi^{*}$ state, but in the low-energy region where it is more intense. Therefore, it is possible that, if it is sufficiently populated, $n \pi^{*}$ can actually contribute to the spectral signal at low energies.

As for thymine, inclusion of solvent effect has very little impact on the computed ESA. The most significant difference between the spectra computed in chloroform and in the gas phase is the small red-shift of the lowest energy peak, due to the slight destabilization of the lowest energy $\pi \pi^{*}$ state in chloroform. Moreover, in solution the most prominent peak, at $\sim 3.5 \mathrm{eV}$, is better resolved.

\subsection{Adenine}

We have analysed the 9- $\mathrm{H}$ tautomer of Adenine, since it is the most stable even in gas-phase, ${ }^{43}$ and is the species present in DNA.

In the OPA spectrum (see Figure 6), EOM-CC3, EOM-CCSD and TD-CAM-B3LYP predict one intense peak falling at $\sim 5.3 \mathrm{eV}$, a more intense one at $\sim 6.5 \mathrm{eV}$, and a very broad absorption in the $7 \sim 8 \mathrm{eV}$ region. These predictions appear in good agreement with the available experimental results, (see the spectra collected in ref. 13) but for an uniform blue-shift of $\sim 0.3 \mathrm{eV}$, mainly due to the lack of vibronic effects in our calculations. The lowest energy band is due to two $\pi \pi^{*}$ transitions usually labelled as $\mathrm{L}_{a}$ and $\mathrm{L}_{b}$, according to the Platt nomenclature. The former, more intense, has a predominant $\mathrm{HOMO} \rightarrow$ LUMO character, the latter, rather weak, a more significant $\mathrm{HOMO} \rightarrow \mathrm{LUMO}+1$ contribution. ${ }^{26}$ Additionally, there is a close lying $n \pi^{*}$ state, which corresponds to a transition from the $\mathrm{N} 1$ and $\mathrm{N} 3$ lone pairs to the LUMO $\pi^{*}$ orbital. $^{26}$ Confirming previous studies, ${ }^{26,52,53}$ at the TD-CAMB3LYP level, $\mathrm{L}_{a}$ is more stable than $\mathrm{L}_{b}$, whereas EOMCCSD and EOM-CC3 provide the opposite trend. However, assessing the exact energy ordering between $\mathrm{L}_{a}$ and $\mathrm{L}_{b}$, which are strongly vibronically coupled, ${ }^{43}$ is not relevant for the present study, as we shall compute the ESA of both states.

Also for adenine, inclusion of solvent effects by PCM has a modest effect on the computed OPA. We observe a small red-shift of the lowest energy bands and the 'usual' increase in the intensity. The $\mathrm{n} \pi^{*}$ state is confirmed to be destabilized in chloroform, by $\sim 0.25 \mathrm{eV}$, less than what observed for the pyrimidines.

We start our analysis of the ESA for the $\mathrm{L}_{b}$ state, the lowest energy one at the EOM-CCSD/CC3 level. Due to the large computational cost, the EOM-CC3 spectrum is limited to the 20 lowest energy states. As a consequence, we limit our discussion to the 0-2 eV energy window. Here, EOM-CC3 and CAM-B3LYP spectra are similar, with a first band peaking just above $1 \mathrm{eV}$, followed by a peak, slightly more intense, at $1.5 \mathrm{eV}$. The EOM-CCSD spec- 
trum is also similar, but for a blue-shift of the two peaks and for the inversion of their relative intensity. At higher energy, both EOM-CCSD and TD-CAM-B3LYP provide a broad absorption band between 2 and $3 \mathrm{eV}$, with two main peaks at $\sim 2.3$ and $\sim 2.7 \mathrm{eV}$. In the investigated energy range, these spectra are consistent with those obtained at the RASPT2 level, ${ }^{13}$ see Figure S4.

Concerning the absorption from $\mathrm{L}_{a}$, EOM-CC3 and TD-CAMB3LYP predict extremely close spectra, but for a small blue-shift of the latter. An intense band appears between 1 and $2 \mathrm{eV}$, peaking at $\sim 1.4 \mathrm{eV}$, with a shoulder at $1.7 \mathrm{eV}$ and a very long tail in the red. Then shallow absorption in the $2 \sim 3 \mathrm{eV}$ energy range is predicted by TD-CAM-B3LYP. The EOM-CCSD spectrum is quite similar, but the relative intensity of the lowest energy main peaks is reverted with respect to the predictions of EOM-CC3 and CAMB3LYP. Significant absorption is then predicted between 2 and 3 $\mathrm{eV}$, with a prominent peak around $2.5 \mathrm{eV}$, not obtained by TDCAM-B3LYP.

The spectra in Figure 7 are in good agreement with the ESA computed in this energy window at the RASPT2 level, which for $\mathrm{L}_{a}$ predicts a strong peak just above $1 \mathrm{eV}$, and for $\mathrm{L}_{b}$ three bands of increasing intensity at $\sim 1,2$, and $2.5 \mathrm{eV}^{13}$

EOM-CC3, EOM-CCSD and TD-CAM-B3LYP agree in yielding a rather substantial ESA also for the $\mathrm{n} \pi^{*}$ state. All the computed spectra show a first band peaking at $\sim 1 \mathrm{eV}$, with a long redwing, and then several peaks between 1.5 and $2 \mathrm{eV}$, giving rise to a band, broader according to CAM-B3LYP, peaking at $\sim 1.8 \mathrm{eV}$. Then, CAM-B3LYP yields a very intense peak a $2.5 \mathrm{eV}$, which is, at least partially, also present at the EOM-CCSD level.

Inclusion of solvent effect has a very limited impact on the computed ESA. Besides the small increase of the intensity, we observe a slight blue-shift of the most intense band of $\mathrm{L}_{a}$, with the peaks present in the gas phase almost coalescing in a single one.

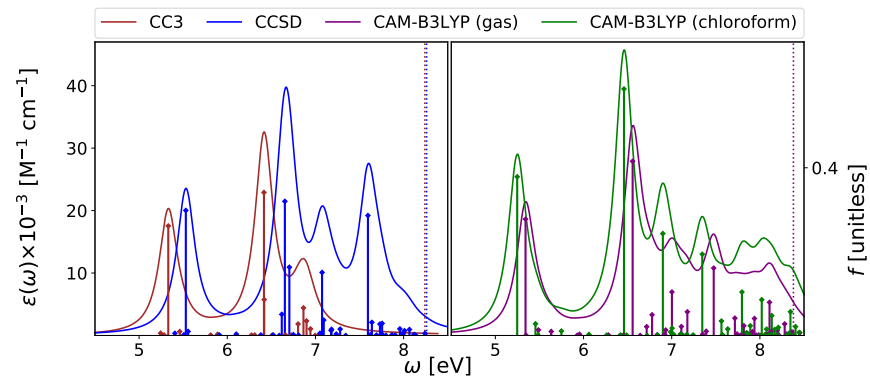

Fig. 6 Adenine. Left: OPA at EOM-CCSD/aug-cc-pVDZ and EOM-CC3/aug-cc-pVDZ levels in gas phase. Right: OPA at CAMB3LYP/aug-cc-pVDZ level of theory in gas phase and in chloroform solution. The vertical lines indicate the first ionisation energy in the gas phase ( $\triangle S C F$ at DFT level). Note that for CAM-B3LYP in gas phase the first intense peak is $\mathrm{L}_{a}$, with $n \pi^{*}$ almost overlapping with it. Then $\mathrm{L}_{b}$ follows. In solution, the first intense peak is $L_{a}$, followed by $L_{b}$ and then by $n \pi^{*}$

\subsection{Guanine}

We focus our analysis on the 9-H tautomer, which is the one present in the DNA. On the other hand, as discussed in a recent study, ${ }^{43}$ in the comparison with experimental spectra obtained in

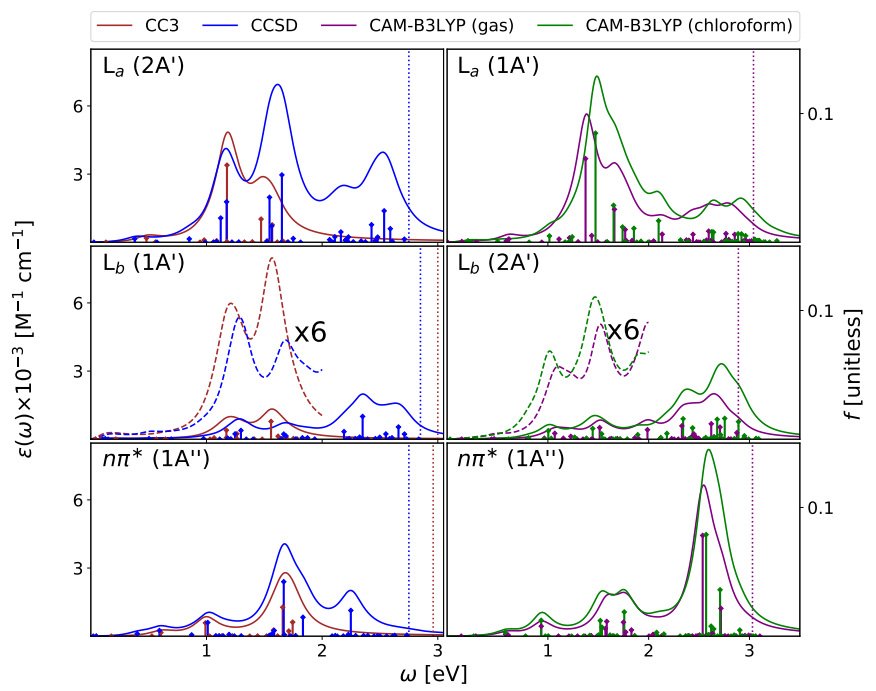

Fig. 7 Adenine. Left: ESA at EOM-CCSD/aug-cc-pVDZ and EOMCC3/aug-cc-pVDZ levels in gas phase. Right: ESA at CAM-B3LYP/augcc-pVDZ level of theory in gas phase and in chloroform solution. The spectra in dashed line were enhanced by the factors indicated in the figures. The vertical lines indicate the first ionisation energy in the gas phase ( $\triangle \mathrm{SCF}$ at DFT level). It was assumed that EOM-CC3 provides the same order of states as EOM-CCSD

the gas phase, the contribution of the 7-H tautomer should be considered. ${ }^{43}$ Due to the size and the large number of excited states of guanine, our EOM-CC3 and EOM-CCSD analysis for OPA is limited to the lowest energy $6.7 \mathrm{eV}$ and $7.8 \mathrm{eV}$, respectively, while the ESA spectra cover only the $0 \sim 2.5 \mathrm{eV}$ range.

In agreement with previous studies, ${ }^{26}$ including the RASPT2 one, ${ }^{13}$ for the $9-\mathrm{H}$ tautomer, TD-CAM-B3LYP and EOM-CC3 predict a strong absorption band above $5 \mathrm{eV}$, with two peaks at 5.0 5.1 eV and at $5.5 \mathrm{eV}$, the most intense one. The EOM-CCSD spectrum is more intense and slightly blue-shifted with respect to the EOM-CC3/TD-CAM-B3LYP ones. Then another intense multipeaked band is found above $7 \mathrm{eV}$, according to both TD-CAMB3LYP and EOM-CCSD.

The lowest energy band is due to two bright $\pi \pi^{*}$ transitions, which, as for Adenine, are usually labelled as $\mathrm{L}_{a}$ and $\mathrm{L}_{b}$. For guanine, however, $\mathrm{L}_{b}$ is twice as intense as $\mathrm{L}_{a}$. As already discussed, ${ }^{43,54}$ the lowest energy dark excited state is a mixed $\pi \sigma^{*} /$ Rydberg transition, which corresponds to $S_{1}$ in the gas phase and to $S_{2}$ in chloroform. For consistency with the other bases, we instead focus on the lowest energy $n \pi^{*}$ state, which involves an excitation from the oxygen lone pair to the $\pi^{*}$ LUMO and it is almost isoenergetic with $\mathrm{L}_{b}$ in the gas phase.

According to EOM-CC3, EOM-CCSD, and TD-CAM-B3LYP the first peak in the ESA spectrum of $\mathrm{L}_{a}$ (see Figure 9) falls at $\sim 0.5$ $\mathrm{eV}$ and corresponds to the $\mathrm{L}_{a} \rightarrow \mathrm{L}_{b}$ transition. The three methods also agree in predicting two additional fairly intense transitions, of similar intensity, the $1 \sim 2 \mathrm{eV}$ spectral range. At $2.4 \sim 2.5 \mathrm{eV}$ both EOM-CCSD and TD-CAM-B3LYP provide an intense band, followed, accroding to CAM-B3LYP of an even stronger band above $3 \mathrm{eV}$. These spectra are similar to those predicted by RASPT2, ${ }^{13}$ 
but for small energy shifts and changes in the relative intensity of the transitions in the range $1.5 \sim 2.5 \mathrm{eV}$.

The most intense $\mathrm{L}_{b}$ ESA peak below $3 \mathrm{eV}$ falls instead at $\sim 1 \mathrm{eV}$, according to EOM-CC3, EOM-CCSD and TD-CAM-B3LYP. The latter method then predicts two other intense peaks just below and above $2 \mathrm{eV}$. RASPT2 also predicts a strong band centered around $1 \mathrm{eV}$, but no strong peak is then found until $3 \mathrm{eV}$ (i.e. the rather strong transitions around $2 \mathrm{eV}$ are missing), ${ }^{13}$ see Figure S5.

Finally, the ESA spectrum of the lowest energy $n \pi^{*}$ state is very rich, but rather weak (see Figure 9), with EOM-CC3, EOM-CCSD and TD-CAM-B3LYP methods providing fairly similar spectral patterns in the low-energy region. A first band is predicted just below $1 \mathrm{eV}$ and a second one, more intense, at $\sim 1.5 \mathrm{eV}$ (according to CAM-B3LYP) and $\sim 1.8 \mathrm{eV}$ (according to EOM-CCSD).

In the high energy part of the spectrum, the TD-CAM-B3LYP method predicts a band at $\sim 2.7 \mathrm{eV}$.

The qualitative trends associated to the inclusion of solvent effects are the same discussed until now: a general increase in the intensity, and $\sim 0.25 \mathrm{eV}$ blue-shift of the lowest-energy $\mathrm{n} \pi^{*}$ state. Interestingly, the ESA spectrum computed in solution for this latter state is significantly more intense than in the gas phase. For guanine some additional transitions also appear in the blue-wing. However, this is likely due to the stabilization of some excited states associated to fairly intense transitions that 'enter' among the excited states considered when computing the spectrum.

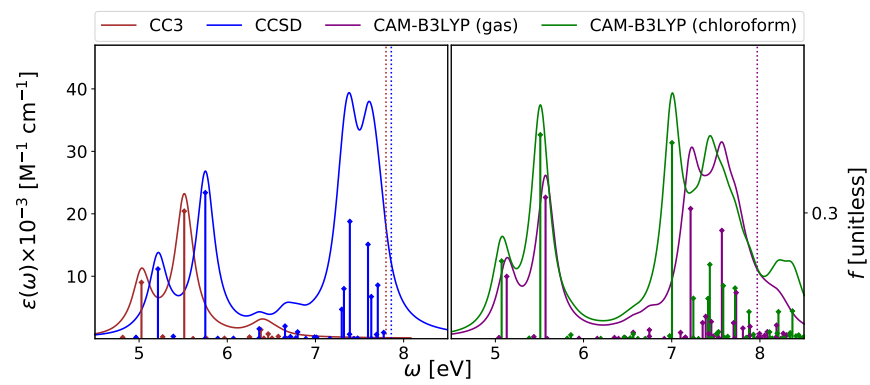

Fig. 8 Guanine. Left: OPA at EOM-CCSD/aug-cc-pVDZ and EOM-CC3/aug-cc-pVDZ levels in gas phase. Right: OPA at CAMB3LYP/aug-cc-pVDZ level of theory in gas phase and in chloroform solution. The vertical dashed lines indicate the first ionisation energy in the gas phase ( $\triangle \mathrm{SCF}$ at DFT level).

\subsection{ESA of $\pi \pi^{*}$ at $\pi \pi^{*}$-minima}

As final step of our analysis, we have computed the ESA from the planar pseudo-minima of the lowest-energy $\pi \pi^{*}$ bright states, which are expected to play a major role in determining the spectral signal. For adenine and guanine, we focused on the $\mathrm{L}_{a}$ minimum, which, independently of the predictions concerning the relative stability in the $\mathrm{FC}$ region with respect to $\mathrm{L}_{b}$, is associated to the lowest energy minimum, according to the vast majority of electronic structure methods. ${ }^{26}$ Unfortunately, a direct comparison with experiments is not easy. The excited state lifetime of the bright states of nucleobases in chloroform is ultrashort ( $\leq 1 \mathrm{ps)},{ }^{55-57}$ since the path from the FC point to the lowest energy Conical Intersection (CoI) with the ground state $S_{0}$ is characterized by a very small, or vanishing energy barrier. ${ }^{26}$ As a con-

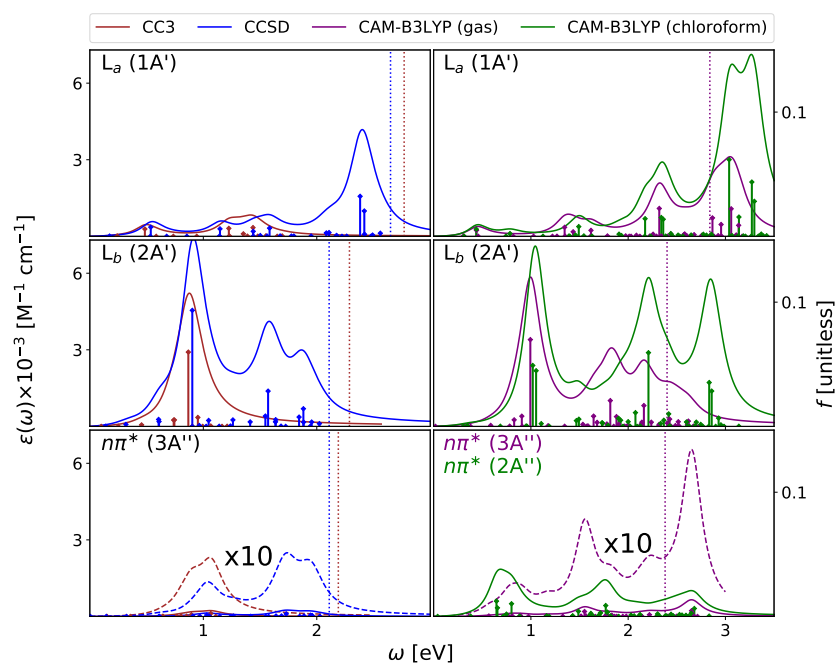

Fig. 9 Guanine. Left: ESA at EOM-CCSD/aug-cc-pVDZ and EOMCC3/aug-cc-pVDZ levels in gas phase. Right: ESA at CAM-B3LYP/augcc-pVDZ level of theory in gas phase and in chloroform solution. The spectra in dashed line are enhanced by the factor indicated in the figures. The vertical lines indicate the first ionisation energy in the gas phase $(\triangle \mathrm{SCF}$ at DFT level).

sequence, the contribution of the ESA in the FC region could be non negligible. On the other side, the path towards the CoI involves a severe distortion of the ring planarity. ${ }^{26,58}$ As discussed in our previous contribution, ${ }^{15}$ such movement leads to a significant mixing between $\pi \pi^{*}$ and $n \pi^{*}$ states. However, due to the absence of a large energy barrier, the photo-excited wave-packet is expected to rapidly pass through the non planar region of the potential energy surface (PES), whose contribution to the signal might be indeed small, also considering that it should be characterized by lower oscillator strengths. A full characterization of the ESA along the decay path from the FC point to the CoI is beyond the scopes of the present article. Instead, in order to get preliminary insights on how the ESA should change in the ultrafast time-regime, where the system approximately preserves a planar configuration, we here optimized the excited-state minima in $\mathrm{C}_{s}$ symmetry. This choice also allows an easier discrimination of the signals expected from $\pi \pi^{*}$ and $\mathrm{n} \pi^{*}$ states.

Before starting our comparison with the experiments, some additional warnings are in order. The available experimental spectra are transient absorption spectra (TAS), ${ }^{55,56}$ which monitor ultrafast dynamical processes, involving several excited states, where the role of vibronic effects and even that of the characteristics of the laser fields (time duration, central frequency, shape) are very important. Moreover, the experiments were carried out on bulky nucleoside derivatives, ${ }^{55,56}$ whereas we here study the bare nucleobase. Finally, the experimental spectra are affected by additional processes, as ground state bleaching and stimulated emission, that are not considered in our calculations, and could mask the ESA in the high energy region, i.e. at $\lambda<350 \mathrm{~nm}$. Notwithstanding these caveats, these experiments can provide a meaningful test of the accuracy of our predictions. The computed spectra 
exhibit an extremely small dependence on the solvation regime (equilibrium vs. non-equilibrium) ${ }^{25,47,59}$ used in the PCM/TDCAM-B3LYP calculations (see ESI, Figure S12).

The computed spectrum of thymine shows a first small peak at $\sim 1.2 \mathrm{eV}(\sim 1000 \mathrm{~nm})$, followed by a band, covering the range $1.5 \sim 2.5 \mathrm{eV}(800-500 \mathrm{~nm})$ and peaking just below $2 \mathrm{eV}(\sim 650$ $\mathrm{nm})$. We then find a shoulder just above $3 \mathrm{eV}(\sim 400 \mathrm{~nm})$, preceding a very intense ESA band at higher energy. The computed spectrum is in nice agreement with the experimental one, available in the range $350-700 \mathrm{~nm}$, which exhibits a band at $\sim 400 \mathrm{~nm}$, preceding a broad band peaking at $\sim 700 \mathrm{~nm} .{ }^{56}$

For cytosine, our calculations predict a rather weak and broad absorption between $1 \mathrm{eV}$ and $2.5 \mathrm{eV}$, with a first band peaking at $0.9 \mathrm{eV}(1400 \mathrm{~nm})$, followed by a band starting above $2 \mathrm{eV}$ $(\sim 620 \mathrm{~nm})$ and peaking at $\sim 2.5 \mathrm{eV}(500 \mathrm{~nm})$. Then, two strong bands are obtained at $3.2 \mathrm{eV}(400 \mathrm{~nm})$ and $4.5 \mathrm{eV}(275 \mathrm{~nm})$. Our predictions seem consistent with the available experimental data, which cover the range between 350 and $700 \mathrm{~nm}$, considering that cytosine emits at $350 \mathrm{~nm}$, and therefore a comparison with our ESA is difficult in that region. In the experimental spectrum, ${ }^{55}$ a weak and broad band, with shallow maxima at $\sim 500$ and $\sim 600$ $\mathrm{nm}$ is indeed present. ${ }^{55}$

The most prominent peak in the spectrum computed for adenine falls at $\sim 1.8 \mathrm{eV}(690 \mathrm{~nm})$ and is followed by another peak at $\sim 2.2 \mathrm{eV}(560 \mathrm{~nm})$. At higher energies, a broad band peaking above $3.0 \mathrm{eV}(\sim 410 \mathrm{~nm})$ is found. Also in this case, the computed spectrum is consistent with the available transient absorption spectrum, which features a very broad band with a maximum at $700 \mathrm{~nm}$, and a peak at $\sim 400 \mathrm{~nm} .{ }^{56}$ Interestingly, we correctly predict the higher ESA intensity of adenine with respect to thymine. On the other hand, the intensity of the $400 \mathrm{~nm}$ feature is underestimated by our calculations.

Finally, for guanine we predict, below $3 \mathrm{eV}$, three bands of increasing intensity, peaking at $\sim 0.5 \mathrm{eV}, \sim 1.8$ and $\sim 2.6 \mathrm{eV}$ (480 $\mathrm{nm})$. They are followed by an intense peak at $3.5 \mathrm{eV}(350 \mathrm{~nm})$. In the experimental spectrum we indeed find a broad band in the 400-620 region, with a maximum at $\sim 480 \mathrm{~nm}$. Moreover, there is a very strong ESA band at $\sim 350 \mathrm{~nm}$, which also agrees with our prediction. ${ }^{55}$ We should again recall that in principle, in this region, transient absorption spectra are also affected by the stimulated emission (SE) signal. The good agreement with ESA computations thus suggests that $\mathrm{SE}$ is rather weak.

\section{Concluding remarks}

We have carried out a thorough exploration of the absorption spectra of the four DNA bases, in the gas phase and in the lowpolarity solvent chloroform, resorting to three single reference quantum mechanical methods: EOM-CC3, EOM-CCSD and TDCAM-B3LYP. Our main focus was the calculation of the ESA spectra, which is fundamental for the assignment and the interpretation of the pump and probe spectra.

The three investigated methods provide similar shapes for the OPA spectra, which are also in good agreement with those obtained with other multi-reference QM methods. ${ }^{13,26}$ As a rule of thumb, EOM-CCSD spectra are, more or less uniformly, blueshifted by $0.2 \sim 0.3 \mathrm{eV}$ with respect to the EOM-CC3 ones, which

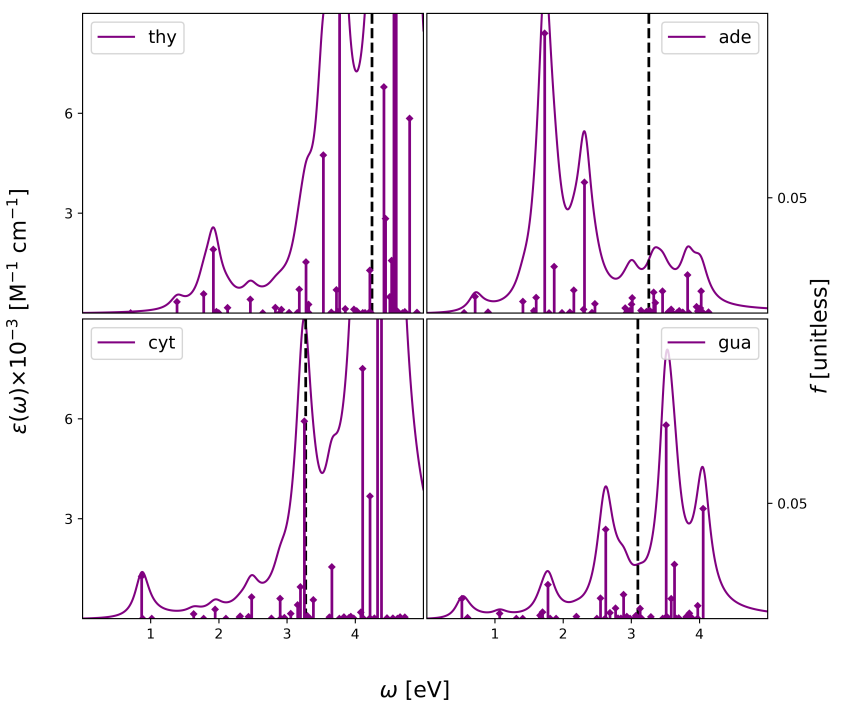

Fig. 10 CAMB3LYP/aug-cc-pVDZ ESA in chloroform solution (equilibrium $\mathrm{PCM}$ ) from the $\pi \pi^{*}$ state at the $\pi \pi^{*}$-minimum (gas phase structure) of all four nucleobases. A vertical dashed line indicates the estimated value of the first ionization energy in the excited state, obtained as IE of the ground state minus the energy of $\pi \pi^{*}$ state.

are very close to the CAM-B3LYP ones. The largest quantitative discrepancy between EOM-CC3 and CAM-B3LYP OPA spectra is found for cytosine and it is smaller than $0.25 \mathrm{eV}$. The spectra are consistent with the available experiments, considering that for guanine and cytosine different tautomers contribute to the gas phase experimental spectra ${ }^{43}$ and that vibronic effects, which are not included in our study, would lead to a systematic red-shift of the computed spectra. ${ }^{45}$

The ESA spectra computed in the gas phase by EOM-CC3, EOMCCSD, and TD-CAM-B3LYP are also in nice agreement, for both $\pi \pi^{*}$ and $\mathrm{n} \pi^{*}$ states. In particular, EOM-CC3 and TD-CAM-B3LYP are, in general, quite close, most of the predicted peaks being within $0.1 \mathrm{eV}$. The predicted spectral shapes are also consistent with the RASPT2 results, which are available for $\pi \pi^{*}$ states. ${ }^{13}$ This result, together with the similarity between EOM-CC3 and EOM-CCSD results, indicate that the role of double excited states is rather limited, at least in the investigate energy window (0$3.5 \mathrm{eV}$ ). The ESA spectra of the investigated $\mathrm{n} \pi^{*}$ states, are, in general, less intense than those of the $\pi \pi^{*}$ states, but, interestingly, their contribution cannot be safely neglected. Moreover, it is worth to remind that all the structures we have investigated are planar and, as a consequence, the $\pi \pi^{*}$ and $n \pi^{*}$ states are decoupled by symmetry. For non planar structures, populated already in the FC region due to thermal fluctuations and even more important in the path leading to the lowest energy CoI with $\mathrm{S}_{0}$, the coupling between the different excited states would be larger, further increasing the computational burden of the calculations. In this respect, the present data provide an important validation of TDCAM-B3LYP, which yields spectra very close to those of EOM-CC3 and in good agreement with the RASPT2 ones. This outcome can pave the way to the study of larger oligonucleotides, which, at the 
moment, can only be tackled at the TD-DFT level. Already for a dinucleotide, the number of excited states to be considered, even in a small energy window, strongly increases, making brute force approaches unfeasible for wavefunction-based QM methods, On the other hand, as discussed above, the use of purposely tailored procedures (e.g., a 'wise' selection of the active space) is more difficult for non 'symmetric structures' and could it make difficult to obtain a well-balanced description in all the regions of the PES. Yet, very encouraging results on the treatment of larger systems, including solvated systems, with CC accuracy come from the latest advances in multilevel coupled cluster theory. ${ }^{60-63}$

Another interesting feature of TD-CAM-B3LYP is the relatively small dependence of the computed spectra on the size of the basis set. As shown in the SI, the spectra obtained at the TDCAM-B3LYP/6-31G(d) level are fairly similar to the ones reported here, but for a moderate, almost uniform, blue-shift. It is, however, clear that a small basis set would make impossible the study of Rydberg transitions and, in general, additional tests may be important to definitively assess the reliability of TD-CAM-B3LYP. Once validated the accuracy of TD-CAM-B3LYP/aug-cc-pvdz calculations in the gas phase, we have exploited this method in the subsequent part of our study, focused on chloroform solution. Based on our experience, ${ }^{59}$ a continuum model as PCM should be sufficient to reproduce solvent effect in such non hydrogenbonding solvent. Inclusion of solvent effect has a rather small, though visible, effect on the spectra. The spectral shapes are similar to those computed in the gas phase and the peaks are only slightly shifted (usually $\leq 0.1 \mathrm{eV}$ ). On the other hand, the lowest energy $\mathrm{n} \pi^{*}$ states are relatively destabilized in chloroform by $0.3 \sim 0.4 \mathrm{eV}$ with respect the bright $\pi \pi^{*}$ states. As a consequence, solvent could have an important 'indirect' impact on the computed TAS, by simply modifying the population transfer between the bright and dark excited states.

In the last part of our analysis, we have computed, for all the four DNA bases, the ESA from the minima of the lowest energy $\pi \pi^{*}$ states in order to compare our predictions with the available TAS spectra in chloroform. Always keeping in mind all the caveats discussed in the preceding subsection, our computed spectra are fully consistent with the experimental ones, for what concerns the position and the relative intensity of the large majority of the peaks. Taken together, the data reported here provide very encouraging indications on the possibility of computing and assigning the ESA spectra of medium size molecules, not only in the gas phase but also in solution. It is clear that many challenges are still ahead (inclusion of vibronic effects, of explicit solute-solvent interactions, direct simulation of TAS spectra, just to name a few). Moreover, additional benchmark tests would be desirable, for what concerns especially the high energy region, where it could not be possible to discard the role of double excited states. However, it seems that, at least in the visible, the computation of the ESA of oligonucleotides in solution is now at hand.

\section{Confirming Author Contributions}

R.I., F.S., and S.C. conceptualized and supervised the project. D.A.F. carried out the TDA and TDDFT calculations and prepared all graphical material. S.C. performed the MOM-TDA calculations. A.C.P. and H.K. performed the EOM-CCSD and EOM-CC3 calculations. R.I., F.S., and S.C. prepared the first draft of the manuscript. All authors discussed the science and revised the article to its final form.

\section{Conflicts of interest}

There are no conflicts to declare.

\section{Acknowledgements}

The authors thank Dr. Vishal Kumar Jaiswal, Dr. Ivan Rivalta and Dr. Marco Garavelli (University of Bologna) for giving us access to the full set of RASPT2 ESA data from ref. 13. S.C. thanks Dr. Andrew Gilbert (Q-Chem) for discussion. This work has received funding from the European Union's Horizon 2020 research and innovation program under the Marie Skłodowska-Curie European Training Network [grant agreement no. 765739 (COSINECOmputational Spectroscopy In Natural sciences and Engineering, D.A.F., A.C.P., H.K., and S.C.) and no. 765266 (LightDyNAmics, R.I. and F.S.). S.C. acknowledges financial support from the Independent Research Fund Denmark-DFF-FNU RP2 (grant no. 7014-00258B). A.C.P., S.C., and H.K. acknowledge the Research Council of Norway through FRINATEK projects 263110 and 275506.

\section{Notes and references}

1 M. Maiuri, M. Garavelli and G. Cerullo, J. Am. Chem. Soc., 2020, 142, 3-15.

2 T. A. A. Oliver, R. Soc. Open Sci., 2018, 5, 171425.

3 S. S. K. Raavi and C. Biswas, in Femtosecond Pump-Probe Spectroscopy for Organic Photovoltaic Devices, American Cancer Society, 2019, pp. 1-49.

4 C. Ruckebusch, M. Sliwa, P. Pernot, A. de Juan and R. Tauler, J. Photochem. Photobiol. C, 2012, 13, 1-27.

5 P. Cronstrand, O. Christiansen, P. Norman and H. Ågren, Phys. Chem. Chem. Phys., 2000, 2, 5357-5363.

6 S. Ling, S. Schumacher, I. Galbraith and M. Paterson, J. Phys. Chem. C, 2013, 117, 6889-6895.

7 J.-C. Denis, A. Ruseckas, G. J. Hedley, A. B. Matheson, M. J. Paterson, G. A. Turnbull, I. D. W. Samuel and I. Galbraith, Phys. Chem. Chem. Phys., 2016, 18, 21937-21948.

8 D. N. Bowman, J. C. Asher, S. A. Fischer, C. J. Cramer and N. Govind, Phys. Chem. Chem. Phys., 2017, 19, 27452 27462.

9 A. Giussani, J. Segarra-Martí, A. Nenov, I. Rivalta, A. Tolomelli, S. Mukamel and M. Garavelli, Theor. Chem. Acc., 2016, 135, 121.

10 A. J. Pepino, J. Segarra-Martí, A. Nenov, R. Improta and M. Garavelli, J. Phys. Chem. Lett., 2017, 8, 1777-1783.

11 L. Martínez-Fernández, A. J. Pepino, J. Segarra-Martí, J. Jovaišaitè, I. Vaya, A. Nenov, D. Markovitsi, T. Gustavsson, A. Banyasz, M. Garavelli and R. Improta, J. Am. Chem. Soc., 2017, 139, 7780-7791.

12 M. Schmid, L. Martinez-Fernandez, D. Markovitsi, F. Santoro, 
F. Hache, R. Improta and P. Changenet, J. Phys. Chem. Lett., 2019, 10, 4089-4094.

13 V. K. Jaiswal, J. Segarra-Martí, M. Marazzi, E. Zvereva, X. Assfeld, A. Monari, M. Garavelli and I. Rivalta, Phys. Chem. Chem. Phys., 2020, 22, 15496-15508.

14 L. González, D. Escudero and L. Serrano-Andrés, ChemPhysChem, 2012, 13, 28-51.

15 D. A. Fedotov, A. C. Paul, P. Posocco, F. Santoro, M. Garavelli, H. Koch, S. Coriani and R. Improta, J. Chem. Theory Comput., 2021, 17, 1638-1652.

16 M. J. G. Peach, P. Benfield, T. Helgaker and D. J. Tozer, J. Chem. Phys., 2008, 128, 044118.

17 J. F. Stanton and R. J. Bartlett, J. Chem. Phys., 1993, 98, 7029-7039.

18 A. C. Paul, R. H. Myhre and H. Koch, J. Chem. Theory Comput., 2021, 0, 0.

19 A. Dreuw and M. Wormit, Wiley Interdiscip. Rev. Comput. Mol. Sci., 2015, 5, 82-95.

20 A. T. B. Gilbert, N. A. Besley and P. M. W. Gill, J. Phys. Chem. A, 2008, 112, 13164-13171.

21 A. Bhattacherjee, C. D. Pemmaraju, K. Schnorr, A. R. Attar and S. R. Leone, J. Am. Chem. Soc., 2017, 139, 16576-16583.

22 T. Northey, J. Norell, A. E. A. Fouda, N. A. Besley, M. Odelius and T. J. Penfold, Phys. Chem. Chem. Phys., 2020, 22, 26672676.

23 S. Tsuru, M. L. Vidal, M. Pápai, A. I. Krylov, K. B. Møller and S. Coriani, Struct. Dyn., 2021, 8, 024101.

24 V. Scutelnic, S. Tsuru, M. Pápai, Z. Yang, M. Epshtein, T. Xue, E. Haugen, Y. Kobayashi, A. I. Krylov, K. B. Møller, S. Coriani and S. R. Leone, Nat. Commun., 2021, 12, 5003.

25 J. Tomasi, B. Mennucci and R. Cammi, Chem. Rev., 2005, 105, 2999.

26 R. Improta, F. Santoro and L. Blancafort, Chem. Rev., 2016, 116, 3540-3593.

27 J. S. Taylor, Acc. Chem. Res., 1994, 27, 76-82.

28 J. Cadet, T. Douki and J.-L. Ravanat, Photochem. Photobiol., 2015, 91, 140-155.

29 T. Douki, Photochem. Photobiol. Sci., 2013, 12, 1286-1302.

30 C. E. Crespo-Hernández, B. Cohen, P. M. Hare and B. Kohler, Chem. Rev., 2004, 104, 1977-2019.

31 K. Kleinermanns, D. Nachtigallová and M. S. de Vries, Int. Rev. Phys. Chem., 2013, 32, 308-342.

32 C. T. Middleton, K. de La Harpe, C. Su, Y. K. Law, C. E. CrespoHernández and B. Kohler, Annu. Rev. Phys. Chem., 2009, 60, 217-239.

33 M. Barbatti, A. C. Borin and S. Ullrich, in Photoinduced Phenomena in Nucleic Acids, ed. M. Barbatti, C. A. Borin and S. Ullrich, Springer International Publishing: Cham, Switzerland, 2015, vol. 355, pp. 1-32.

34 S. Mai, M. Richter, P. Marquetand and L. González, Photoinduced Phenomena in Nucleic Acids I: Nucleobases in the Gas Phase and in Solvents, 2015, pp. 99-153.

35 W. J. Schreier, P. Gilch and W. Zinth, Ann. Rev. Phys. Chem., 2015, 66, 497-519.
36 K. Aidas, C. Angeli, K. L. Bak, V. Bakken, R. Bast, L. Boman, O. Christiansen, R. Cimiraglia, S. Coriani, P. Dahle, E. K. Dalskov, U. Ekström, T. Enevoldsen, J. J. Eriksen, P. Ettenhuber, B. Fernández, L. Ferrighi, H. Fliegl, L. Frediani, K. Hald, A. Halkier, C. Hättig, H. Heiberg, T. Helgaker, A. C. Hennum, H. Hettema, E. Hjertenæs, S. Høst, I.-M. Høyvik, M. F. Iozzi, B. Jansik, H. J. A. Jensen, D. Jonsson, P. Jørgensen, J. Kauczor, S. Kirpekar, T. Kjærgaard, W. Klopper, S. Knecht, R. Kobayashi, H. Koch, J. Kongsted, A. Krapp, K. Kristensen, A. Ligabue, O. B. Lutnæs, J. I. Melo, K. V. Mikkelsen, R. H. Myhre, C. Neiss, C. B. Nielsen, P. Norman, J. Olsen, J. M. H. Olsen, A. Osted, M. J. Packer, F. Pawlowski, T. B. Pedersen, P. F. Provasi, S. Reine, Z. Rinkevicius, T. A. Ruden, K. Ruud, V. V. Rybkin, P. Salek, C. C. M. Samson, A. S. de Merás, T. Saue, S. P. A. Sauer, B. Schimmelpfennig, K. Sneskov, A. H. Steindal, K. O. Sylvester-Hvid, P. R. Taylor, A. M. Teale, E. I. Tellgren, D. P. Tew, A. J. Thorvaldsen, L. Thøgersen, O. Vahtras, M. A. Watson, D. J. D. Wilson, M. Ziolkowski and H. Ågren, WIREs Comput. Mol. Sci., 2014, 4, 269.

37 S. D. Folkestad, E. F. Kjønstad, R. H. Myhre, J. H. Andersen, A. Balbi, S. Coriani, T. Giovannini, L. Goletto, T. S. Haugland, A. Hutcheson, I.-M. Høyvik, T. Moitra, A. C. Paul, M. Scavino, A. S. Skeidsvoll, A. H. Tveten and H. Koch, J. Chem. Phys., 2020, 152, 184103.

38 S. G. Balasubramani, G. P. Chen, S. Coriani, M. Diedenhofen, M. S. Frank, Y. J. Franzke, F. Furche, R. Grotjahn, M. E. Harding, C. Hättig, A. Hellweg, B. Helmich-Paris, C. Holzer, U. Huniar, M. Kaupp, A. Marefat Khah, S. Karbalaei Khani, T. Müller, F. Mack, B. D. Nguyen, S. M. Parker, E. Perlt, D. Rappoport, K. Reiter, S. Roy, M. Rückert, G. Schmitz, M. Sierka, E. Tapavicza, D. P. Tew, C. van Wüllen, V. K. Voora, F. Weigend, A. Wodyński and J. M. Yu, J. Chem. Phys., 2020, 152, 184107.

39 E. Epifanovsky, A. T. B. Gilbert, X. Feng, J. Lee, Y. Mao, N. Mardirossian, P. Pokhilko, A. F. White, M. P. Coons, A. L. Dempwolff, Z. Gan, D. Hait, P. R. Horn, L. D. Jacobson, I. Kaliman, J. Kussmann, A. W. Lange, K. U. Lao, D. S. Levine, J. Liu, S. C. McKenzie, A. F. Morrison, K. D. Nanda, F. Plasser, D. R. Rehn, M. L. Vidal, Z.-Q. You, Y. Zhu, B. Alam, B. J. Albrecht, A. Aldossary, E. Alguire, J. H. Andersen, V. Athavale, D. Barton, K. Begam, A. Behn, N. Bellonzi, Y. A. Bernard, E. J. Berquist, H. G. A. Burton, A. Carreras, K. Carter-Fenk, R. Chakraborty, A. D. Chien, K. D. Closser, V. Cofer-Shabica, S. Dasgupta, M. de Wergifosse, J. Deng, M. Diedenhofen, H. Do, S. Ehlert, P.-T. Fang, S. Fatehi, Q. Feng, T. Friedhoff, J. Gayvert, Q. Ge, G. Gidofalvi, M. Goldey, J. Gomes, C. E. González-Espinoza, S. Gulania, A. O. Gunina, M. W. D. Hanson-Heine, P. H. P. Harbach, A. Hauser, M. F. Herbst, M. Hernández Vera, M. Hodecker, Z. C. Holden, S. Houck, X. Huang, K. Hui, B. C. Huynh, M. Ivanov, Á. Jász, H. Ji, H. Jiang, B. Kaduk, S. Kähler, K. Khistyaev, J. Kim, G. Kis, P. Klunzinger, Z. Koczor-Benda, J. H. Koh, D. Kosenkov, L. Koulias, T. Kowalczyk, C. M. Krauter, K. Kue, A. Kunitsa, T. Kus, I. Ladjánszki, A. Landau, K. V. Lawler, D. Lefrancois, S. Lehtola, R. R. Li, Y.-P. Li, J. Liang, M. Liebenthal, 
H.-H. Lin, Y.-S. Lin, F. Liu, K.-Y. Liu, M. Loipersberger, A. Luenser, A. Manjanath, P. Manohar, E. Mansoor, S. F. Manzer, S.-P. Mao, A. V. Marenich, T. Markovich, S. Mason, S. A. Maurer, P. F. McLaughlin, M. F. S. J. Menger, J.-M. Mewes, S. A. Mewes, P. Morgante, J. W. Mullinax, K. J. Oosterbaan, G. Paran, A. C. Paul, S. K. Paul, F. Pavošević, Z. Pei, S. Prager, E. I. Proynov, Á. Rák, E. Ramos-Cordoba, B. Rana, A. E. Rask, A. Rettig, R. M. Richard, F. Rob, E. Rossomme, T. Scheele, M. Scheurer, M. Schneider, N. Sergueev, S. M. Sharada, W. Skomorowski, D. W. Small, C. J. Stein, Y.-C. Su, E. J. Sundstrom, Z. Tao, J. Thirman, G. J. Tornai, T. Tsuchimochi, N. M. Tubman, S. P. Veccham, O. Vydrov, J. Wenzel, J. Witte, A. Yamada, K. Yao, S. Yeganeh, S. R. Yost, A. Zech, I. Y. Zhang, X. Zhang, Y. Zhang, D. Zuev, A. AspuruGuzik, A. T. Bell, N. A. Besley, K. B. Bravaya, B. R. Brooks, D. Casanova, J.-D. Chai, S. Coriani, C. J. Cramer, G. Cserey, A. E. DePrince, R. A. DiStasio, A. Dreuw, B. D. Dunietz, T. R. Furlani, W. A. Goddard, S. Hammes-Schiffer, T. Head-Gordon, W. J. Hehre, C.-P. Hsu, T.-C. Jagau, Y. Jung, A. Klamt, J. Kong, D. S. Lambrecht, W. Liang, N. J. Mayhall, C. W. McCurdy, J. B. Neaton, C. Ochsenfeld, J. A. Parkhill, R. Peverati, V. A. Rassolov, Y. Shao, L. V. Slipchenko, T. Stauch, R. P. Steele, J. E. Subotnik, A. J. W. Thom, A. Tkatchenko, D. G. Truhlar, T. Van Voorhis, T. A. Wesolowski, K. B. Whaley, H. L. Woodcock, P. M. Zimmerman, S. Faraji, P. M. W. Gill, M. HeadGordon, J. M. Herbert and A. I. Krylov, J. Chem. Phys., 2021, 155, 084801.

40 J. F. Stanton and J. Gauss, J. Chem. Phys., 1999, 111, 87858788.

41 S. Coriani and H. Koch, J. Chem. Phys., 2015, 143, 181103.

42 S. Coriani and H. Koch, J. Chem. Phys., 2016, 145, 149901.

43 J. A. Green, M. Yaghoubi Jouybari, D. Aranda, R. Improta and F. Santoro, Molecules, 2021, 26, 1-23.

44 L. B. Clark, G. G. Peschel and I. Tinoco, J. Phys. Chem., 1965, 69, 3615-3618.

45 F. J. Avila Ferrer, J. Cerezo, E. Stendardo, R. Improta and F. Santoro, J. Chem. Theory Comput., 2013, 9, 2072-2082.

46 R. Improta, V. Barone, G. Scalmani and M. J. Frisch, J. Chem. Phys., 2006, 125, 054103.

47 M. Cossi and V. Barone, J. Chem. Phys., 2001, 115, 4708.

48 R. Improta and V. Barone, in Excited states behavior of $\underline{\text { nucleobases in solution: Insights from computational studies, }}$ ed. M. Barbatti, C. A. Borin and S. Ullrich, Springer International Publishing: Cham, Switzerland, 2015, vol. 355, pp. 329-358.

49 G. Bazsó, G. Tarczay, G. Fogarasi and P. G. Szalay, Phys. Chem. Chem. Phys., 2011, 13, 6799-6807.

50 Y. Liu, L. Martínez Fernández, J. Cerezo, G. Prampolini, R. Improta and F. Santoro, Chem. Phys., 2018, 515, 452-463.

51 M. Yaghoubi Jouybari, Y. Liu, R. Improta and F. Santoro, J. Chem. Theory Comput., 2020, 16, 5792-5808.

52 F. Santoro, R. Improta, T. Fahleson, J. Kauczor, P. Norman and S. Coriani, J. Phys. Chem. Lett., 2014, 5, 1806-1811.

53 S. K. Khani, R. Faber, F. Santoro, C. Hättig and S. Coriani, J. Chem. Theory Comput., 2019, 15, 1242-1254.

54 V. Karunakaran, K. Kleinermanns, R. Improta and S. a. Kovalenko, J. Am. Chem. Soc., 2009, 131, 5839-50.

55 K. Röttger, H. J. B. Marroux, M. P. Grubb, P. M. Coulter, H. Böhnke, A. S. Henderson, M. C. Galan, F. Temps, A. J. Orr-Ewing and G. M. Roberts, Angew. Chem. Int. Ed., 2015, 54, 14719-14722.

56 K. Röttger, H. J. B. Marroux, A. F. M. Chemin, E. Elsdon, T. A. A. Oliver, S. T. G. Street, A. S. Henderson, M. C. Galan, A. J. Orr-Ewing and G. M. Roberts, J. Phys. Chem. B, 2017, 121, 4448-4455.

57 K. Röttger, H. J. B. Marroux, H. Böhnke, D. T. J. Morris, A. T. Voice, F. Temps, G. M. Roberts and A. J. Orr-Ewing, Faraday Discuss., 2016, 194, 683-708.

58 A. Giussani, J. Segarra-Martí, D. Roca-Sanjuán and M. Merchán, Photoinduced Phenomena in Nucleic Acids I: Nucleobases in the Gas Phase and in Solvents, 2015, pp. 55-97.

59 R. Improta, in UV-Visible Absorption and Emission Energies in Condensed Phase by PCM/TD-DFT Methods, John Wiley and Sons, Ltd, 2011, ch. 1, pp. 37-75.

60 R. H. Myhre, A. M. J. Sánchez de Merás and H. Koch, J. Chem. Phys., 2014, 141, 224105.

61 S. D. Folkestad, E. F. Kjønstad, L. Goletto and H. Koch, J. Chem. Theory Comput., 2021, 17, 714-726.

62 L. Goletto, T. Giovannini, S. D. Folkestad and H. Koch, Phys. Chem. Chem. Phys., 2021, 23, 4413-4425.

63 S. D. Folkestad and H. Koch, J. Chem. Theory Comput., 2020, 16, 6869-6879. 